\title{
State estimate based control design using the unified algebraic approach
}

\author{
ANNA FILASOVÁ and DUŠAN KROKAVEC
}

\begin{abstract}
The LMI based method for the control with the state estimate, subject to the input variable constraints in the state feedback control of the linear MIMO systems, is presented in the paper. For this problem are obtained the state feedback as well as the estimator gain matrices that capture the required stability by solving the linear matrix inequalities formulated in the sense of the unified algebraic approach. The method is particularly effective when the input variable constraints and the system output are of interest.
\end{abstract}

Key words: state feedback, linear estimation, linear matrix inequalities, constrains

\section{Introduction}

The trust in the development of observers for linear causal systems came from the introduction of the state-space methods by Kalman [7]. In 1963, Luenberger initiated the theory of observers for the state reconstruction of linear dynamical systems [11] and the observers has come to take that pride of place in the control system design. For linear systems, the corresponding theory was quite established for several years now [15] and large amount of knowledge on designing nonlinear observers and Kalman filters has been accumulated through the literature [1], [17], too. The problem of observer design naturally arise with application of the internal system information obtained from the external measurements, where this need is motivated by many new application purposes, like system monitoring [2], and the control system fault detection [4], [8].

In recent years, linear matrix inequalities (LMI's) have emerged as a powerful tool to approach problems that appear hard if not impossible to solve in an analytical manner [13], [19]. Boosted by the availability of LMI solvers ( [6], [16]), the intention is to reformulate a given problem using sets of LMIs and to optimize functionals over LMI

The Authors are with Department of Cybernetics and Artificial Intelligence, Technical University of Košice, Košice, Slovak Republic, fax: +421 55625 3574, e-mail: anna.filasova@tuke.sk, dusan.krokavec@tuke.sk

The work presented in this paper was supported by VEGA, Grant Agency of Ministry of Education and Academy of Science of Slovak Republic under Grant No. 1/0328/08. This support is very gratefully acknowledged.

Received 30.10.2009. Revised 24.02.2010. 
constraints considering LMI variables. These methods lead to an efficient numerical solution and are particularly suited to problems with uncertain data and multiple (possibly conflicting) specifications.

Linear matrix inequalities (LMI) are now effectively used for synthesis of such controllers that stabilize given systems. Various variants of statements of such problems, including requirements of parameter optimization, can be met in the literature, e.g. in [3], [12], [18].

In this paper the problem of the estimator matrix parameter design for the output limited and the input variable constrained state feedback control is translated into the LMI framework as the extension of idea presented in [10] and solved through the unified algebraic technique to find the controller and estimator gain matrices. The set of the linear matrix inequalities is outlined to possess the necessary conditions for the control parameter design in given estimator-based control structure. The used principle combines the unified algebraic approach and the standard convex-optimization-based LMI algorithms in such a way, that the stable control structure is obtained by solving LMIs which reflect given input variables constraint conditions.

\section{Problem Description}

\subsection{System Model}

Systems under consideration are linear dynamic MIMO systems, represented by the set of the state-space equations

$$
\begin{gathered}
\dot{\boldsymbol{q}}(t)=\boldsymbol{A} \boldsymbol{q}(t)+\boldsymbol{B} \boldsymbol{u}(t) \\
\boldsymbol{y}(t)=\boldsymbol{C} \boldsymbol{q}(t)
\end{gathered}
$$

$\boldsymbol{q}(t) \in \mathbb{R}^{n}, \boldsymbol{u}(t) \in \mathbb{R}^{r}, \boldsymbol{y}(t) \in \mathbb{R}^{m}$ are system state, input and output vectors, respectively, and $\boldsymbol{A} \in \mathbb{R}^{n \times n}, \boldsymbol{B} \in \mathbb{R}^{n \times r}, \boldsymbol{C} \in \mathbb{R}^{m \times n}$ are real matrices. Problem of interest is to design an asymptotically stable closed loop system with the linear memory-less, and in all inputs constrained state feed-back controller of the form

$$
\begin{gathered}
\boldsymbol{u}(t)=-\boldsymbol{K} \boldsymbol{q}_{e}(t) \\
\left|u_{h}(t)\right| \leqslant u_{h m}, \quad h=1,2, \ldots, r
\end{gathered}
$$

where $\boldsymbol{q}_{e}(t) \in \mathbb{R}^{n}$ is an estimate of the system state vector, $u_{h m} \in \mathbb{R}$ is the $h$-th input constraint, and $K \in \mathbb{R}^{r \times n}$ is the feedback controller gain matrix.

To allow the stabilization of (1), (2) using output variable measurement only, the system is extended by Luenberger estimator of the form

$$
\begin{gathered}
\dot{\boldsymbol{q}}_{e}(t)=\boldsymbol{A} \boldsymbol{q}_{e}(t)+\boldsymbol{B} \boldsymbol{u}(t)+\boldsymbol{J}\left(\boldsymbol{y}(t)-\boldsymbol{y}_{e}(t)\right) \\
\boldsymbol{y}_{e}(t)=\boldsymbol{C} \boldsymbol{q}_{e}(t)
\end{gathered}
$$


where $\boldsymbol{J} \in \mathbb{R}^{n \times m}$ is the estimator gain matrix.

Since this formulation under constrained inputs is generally non-convex, using the separability principle the design task will be realized in the two steps. At first a state controller without estimator is solved in the form

$$
\begin{gathered}
\boldsymbol{u}(t)=-\boldsymbol{K} \boldsymbol{q}(t) \\
\left|u_{h}(t)\right| \leqslant u_{h m}, \quad h=1,2, \ldots, r
\end{gathered}
$$

and at the second the estimator is designed to solve the problem of state estimation for the given controller gain matrix.

\subsection{Basic Preliminaries}

Definition 1 (Null space) Let $\boldsymbol{E}, \boldsymbol{E} \in \mathbb{R}^{h \times h}, \operatorname{ran} k(\boldsymbol{E})=k<h$ be a rank deficient matrix. Then the null space $\mathcal{N}$ of $\boldsymbol{E}$ is the orthogonal complement of the row space of $\boldsymbol{E}$.

Lemma 1 (Orthogonal complement) Let $\boldsymbol{E}, \boldsymbol{E} \in \mathbb{R}^{h \times h}, \operatorname{rank}(\boldsymbol{E})=k<h$ be a rank deficient matrix. Then an orthogonal complement $\boldsymbol{E}^{\perp}$ of $\boldsymbol{E}$ is

$$
\boldsymbol{E}^{\perp}=\boldsymbol{D} \boldsymbol{U}_{2}^{T}
$$

where $\boldsymbol{U}_{2}^{T}$ is the null space of $\boldsymbol{E}$ and $\boldsymbol{D}$ is an arbitrary matrix of appropriate dimension.

Proof. (e.g. see [5], [14]) The singular value decomposition (SVD) of $\boldsymbol{E}, \boldsymbol{E} \in \mathbb{R}^{h \times h}$, $\operatorname{rank}(\boldsymbol{E})=k<h$ gives

$$
\boldsymbol{U}^{T} \boldsymbol{E} \boldsymbol{V}=\left[\begin{array}{c}
\boldsymbol{U}_{1}^{T} \\
\boldsymbol{U}_{2}^{T}
\end{array}\right] \boldsymbol{E}\left[\begin{array}{ll}
\boldsymbol{V}_{1} & \boldsymbol{V}_{2}
\end{array}\right]=\left[\begin{array}{ll}
\boldsymbol{\Sigma}_{1} & \mathbf{0}_{12} \\
\mathbf{0}_{21} & \mathbf{0}_{22}
\end{array}\right]
$$

where $U^{T} \in \mathbb{R}^{h \times h}$ is the orthogonal matrix of the left singular vectors, $\mathbb{W} \in \mathbb{R}^{h \times h}$ is the orthogonal matrix of the right singular vectors of $\mathbb{E}$ and $\boldsymbol{\Sigma}_{1} \in \mathbb{R}^{k \times k}$ is the diagonal positive definite matrix of the form

$$
\boldsymbol{\Sigma}_{1}=\left[\begin{array}{lll}
\sigma_{1} & & \\
& \ddots & \\
& & \sigma_{k}
\end{array}\right], \quad \sigma_{1} \geqslant \cdots \geqslant \sigma_{k}>0
$$

which diagonal elements are the singular values of $\boldsymbol{E}$. Using orthogonal properties of $\boldsymbol{U}$ and $\boldsymbol{V}$, i.e. $\boldsymbol{U}^{T} \boldsymbol{U}=\boldsymbol{I}_{h}$, as well as $\boldsymbol{V}^{T} \boldsymbol{V}=\boldsymbol{I}_{h}$, and

$$
\left[\begin{array}{l}
\boldsymbol{U}_{1}^{T} \\
\boldsymbol{U}_{2}^{T}
\end{array}\right]\left[\begin{array}{ll}
\boldsymbol{U}_{1} & \boldsymbol{U}_{2}
\end{array}\right]=\left[\begin{array}{cc}
\boldsymbol{I}_{1} & \mathbf{0} \\
\mathbf{0} & \boldsymbol{I}_{2}
\end{array}\right], \quad \boldsymbol{U}_{2}^{T} \boldsymbol{U}_{1}=\mathbf{0}
$$


respectively, where $\boldsymbol{I}_{(\cdot)}$ is the identity matrix of appropriate dimension, then $\boldsymbol{E}^{\prime}$ can be written as

$$
\boldsymbol{E}=\boldsymbol{U} \boldsymbol{\Sigma} \boldsymbol{V}^{T}=\left[\begin{array}{ll}
\boldsymbol{U}_{1} & \boldsymbol{U}_{2}
\end{array}\right]\left[\begin{array}{ll}
\boldsymbol{\Sigma}_{1} & \mathbf{0}_{12} \\
\mathbf{0}_{21} & \mathbf{0}_{22}
\end{array}\right]\left[\begin{array}{l}
\boldsymbol{V}_{1}^{T} \\
\boldsymbol{V}_{2}^{T}
\end{array}\right]=\left[\begin{array}{ll}
\boldsymbol{U}_{1} & \boldsymbol{U}_{2}
\end{array}\right]\left[\begin{array}{l}
\boldsymbol{S}_{1} \\
\mathbf{0}_{2}
\end{array}\right]=\boldsymbol{U}_{1} \boldsymbol{S}_{1}
$$

where $S_{1}=\Sigma_{1} \boldsymbol{V}_{1}^{T}$. Thus, (12) and (13) implies

$$
\boldsymbol{U}_{2}^{T} \boldsymbol{E}=\boldsymbol{U}_{2}^{T}\left[\begin{array}{ll}
\boldsymbol{U}_{1} & \boldsymbol{U}_{2}
\end{array}\right]\left[\begin{array}{l}
\boldsymbol{S}_{1} \\
\mathbf{0}_{2}
\end{array}\right]=\mathbf{0} .
$$

It is evident that for an arbitrary matrix $\boldsymbol{D}$ is

$$
\begin{gathered}
\boldsymbol{D} \boldsymbol{U}_{2}^{T} \boldsymbol{E}=\boldsymbol{E}^{\perp} \boldsymbol{E}=\mathbf{0} \\
\boldsymbol{E}^{\perp}=\boldsymbol{D} \boldsymbol{U}_{2}^{T}
\end{gathered}
$$

respectively, which implies (9).

Lemma 2 (Schur Complement) Considering matrices $\boldsymbol{Q}=\boldsymbol{Q}^{T}, \boldsymbol{R}=\boldsymbol{R}^{T}, \boldsymbol{S}$ of appropriate dimensions where $\operatorname{det} \boldsymbol{R} \neq 0$, then the following statements are equivalent:

$$
\left[\begin{array}{cc}
\boldsymbol{Q} & \boldsymbol{S} \\
\boldsymbol{S}^{T} & -\boldsymbol{R}
\end{array}\right]<0 \Leftrightarrow\left[\begin{array}{cc}
\boldsymbol{Q}+\boldsymbol{S} \boldsymbol{R}^{-1} \boldsymbol{S}^{T} & 0 \\
\boldsymbol{0} & -\boldsymbol{R}
\end{array}\right]<0 \Leftrightarrow \boldsymbol{Q}+\boldsymbol{S R}^{-1} \boldsymbol{S}^{T}<0, \boldsymbol{R}>0 .
$$

Proof. (e.g. see [3], [9]) Let the linear matrix inequality takes form

$$
\left[\begin{array}{rr}
\boldsymbol{Q} & \boldsymbol{S} \\
\boldsymbol{S}^{T} & -\boldsymbol{R}
\end{array}\right]<0
$$

Thus, using Gauss elimination it yields

$$
\left[\begin{array}{cc}
\boldsymbol{I} & \boldsymbol{S} \boldsymbol{R}^{-1} \\
\mathbf{0} & \boldsymbol{I}
\end{array}\right]\left[\begin{array}{cc}
\boldsymbol{Q} & \boldsymbol{S} \\
\boldsymbol{S}^{T} & -\boldsymbol{R}
\end{array}\right]\left[\begin{array}{cc}
\boldsymbol{I} & \mathbf{0} \\
\boldsymbol{R}^{-1} \boldsymbol{S}^{T} & \boldsymbol{I}
\end{array}\right]=\left[\begin{array}{cc}
\boldsymbol{Q}+\boldsymbol{S} \boldsymbol{R}^{-1} \boldsymbol{S}^{T} & \mathbf{0} \\
\mathbf{0} & -\boldsymbol{R}
\end{array}\right]
$$

where $\boldsymbol{I}$ is the identity matrix of appropriate dimension. Since

$$
\operatorname{det}\left[\begin{array}{cc}
\boldsymbol{I} & \boldsymbol{S} \boldsymbol{R}^{-1} \\
\mathbf{0} & \boldsymbol{I}
\end{array}\right]=1
$$

given transformation doesn't change the negativity of (18), i.e. yields (17). 


\section{State feedback controller design}

Theorem 1 The system (1), (2) under control law (3), constrained in the input variables and limited in the output is stable if there exist scalar $\gamma>0$ and matrices $\mathbb{Y}=\boldsymbol{Y}^{T}>0$, $\boldsymbol{X}$ such that

$$
\begin{aligned}
& {\left[\begin{array}{cc}
\boldsymbol{Y} \boldsymbol{A}^{T}+\boldsymbol{A} \boldsymbol{Y}-\boldsymbol{B X}-\boldsymbol{X}^{T} \boldsymbol{B}^{T} & \boldsymbol{Y} \boldsymbol{C}^{T} \\
* & -\gamma \boldsymbol{I}_{m}
\end{array}\right]<0} \\
& {\left[\begin{array}{ll}
\boldsymbol{Y} & \boldsymbol{X}^{T} \\
* & \boldsymbol{M}_{r}
\end{array}\right]>0}
\end{aligned}
$$

where

$$
\boldsymbol{X}=\boldsymbol{K} \boldsymbol{Y}
$$

$\boldsymbol{I}_{m} \in \mathbb{R}^{m \times m}$ is the identity matrix and $\boldsymbol{M}_{r} \in \mathbb{R}^{r \times r}$ is a slack matrix which diagonal elements are squares of the input variables constraints.

Hereafter, $*$ denotes the symmetric item in a symmetric matrix.

Proof. Since there is given limit on the output, Lyapunov function can be chosen as follows

$$
v(\boldsymbol{q}(t))=\boldsymbol{q}^{T}(t) \boldsymbol{P} \boldsymbol{q}(t)+\int_{0}^{t} \boldsymbol{\gamma}^{-1} \boldsymbol{y}^{T}(r) \boldsymbol{y}(r) d r>0
$$

and $\gamma>0, \boldsymbol{P}=\boldsymbol{P}^{T}>0, \boldsymbol{P} \in \mathbb{R}^{n \times n}$. Evaluating derivative of $v(\boldsymbol{q}(t)$ with respect to $t$ it can be obtained

$$
\dot{v}(\boldsymbol{q}(t))=\dot{\boldsymbol{q}}^{T}(t) \boldsymbol{P} \boldsymbol{q}(t)+\boldsymbol{q}^{T}(t) \boldsymbol{P} \dot{\boldsymbol{q}}(t)+\boldsymbol{\gamma}^{-1} \boldsymbol{y}^{T}(t) \boldsymbol{y}(t)-\boldsymbol{\gamma}^{-1} \boldsymbol{y}^{T}(0) \boldsymbol{y}(0)<0 .
$$

Then the substitution of (1), (2), and (7) in (25) gives result

$$
\begin{gathered}
\dot{v}(\boldsymbol{q}(t))=-\gamma^{-1} \boldsymbol{q}^{T}(0) \boldsymbol{C}^{T} \boldsymbol{C} \boldsymbol{q}(0)+ \\
+\boldsymbol{q}^{T}(t)\left(\boldsymbol{A}^{T} \boldsymbol{P}+\boldsymbol{P} \boldsymbol{A}-\boldsymbol{P} \boldsymbol{B} \boldsymbol{K}-\boldsymbol{K}^{T} \boldsymbol{B}^{T} \boldsymbol{P}\right) \boldsymbol{q}(t)+\boldsymbol{\gamma}^{-1} \boldsymbol{q}^{T}(t) \boldsymbol{C}^{T} \boldsymbol{C} \boldsymbol{q}(t)<0 .
\end{gathered}
$$

The design problem can be cast as a convex optimization problem. Therefore, (26) is guarantied to be fulfilled if the matrix inequality

$$
\boldsymbol{A}^{T} \boldsymbol{P}+\boldsymbol{P A}-\boldsymbol{P} \boldsymbol{B K}-\boldsymbol{K}^{T} \boldsymbol{B}^{T} \boldsymbol{P}+\boldsymbol{\gamma}^{-1} \boldsymbol{C}^{T} \boldsymbol{C}<0
$$

is satisfied. Pre-multiplying left-hand side and right-hand side of (27) by $\mathbb{P}^{-1}$ results in

$$
\boldsymbol{P}^{-1} \boldsymbol{A}^{T}+\boldsymbol{A} \boldsymbol{P}^{-1}-\boldsymbol{B} \boldsymbol{K} \boldsymbol{P}^{-1}-\boldsymbol{P}^{-1} \boldsymbol{K}^{T} \boldsymbol{B}^{T}+\boldsymbol{\gamma}^{-1} \boldsymbol{P}^{-1} \boldsymbol{C}^{T} \boldsymbol{C} \boldsymbol{P}^{-1}<0 .
$$

Thus, using (17) it is

$$
\left[\begin{array}{cc}
\boldsymbol{Y} \boldsymbol{A}^{T}+\boldsymbol{A} \boldsymbol{Y}-\boldsymbol{B} \boldsymbol{X}-\boldsymbol{X}^{T} \boldsymbol{B}^{T} & \boldsymbol{Y} \boldsymbol{C}^{T} \\
* & -\gamma \boldsymbol{I}_{m}
\end{array}\right]<0
$$


where

$$
\boldsymbol{Y}=\boldsymbol{P}^{-1}, \quad \boldsymbol{X}=\boldsymbol{K} \boldsymbol{Y}
$$

which implies (21).

Since the input constraint inequalities given in (8) can be reformulated as

$$
\|\boldsymbol{K} \boldsymbol{q}(t)\|^{2}=\|\boldsymbol{X} \boldsymbol{P} \boldsymbol{q}(t)\|^{2}=\boldsymbol{u}^{T}(t) \boldsymbol{u}(t) \leqslant \boldsymbol{u}_{m}^{T} \boldsymbol{u}_{m}
$$

where $\|\cdot\|$ denotes any vector norm, then using Frobenius norm (31) implies

$$
\begin{aligned}
& \boldsymbol{q}^{T}(t) \boldsymbol{P} \boldsymbol{X}^{T} \boldsymbol{X} \boldsymbol{P} \boldsymbol{q}(t) \leqslant \boldsymbol{u}_{m}^{T} \boldsymbol{u}_{m}=\eta \\
& \boldsymbol{q}^{T}(t) \boldsymbol{P}^{\frac{1}{2}} \boldsymbol{P}^{\frac{1}{2}} \boldsymbol{X}^{T} \boldsymbol{X} \boldsymbol{P}^{\frac{1}{2}} \boldsymbol{P}^{\frac{1}{2}} \boldsymbol{q}(t) \leqslant \eta
\end{aligned}
$$

respectively, where $\eta=\boldsymbol{u}_{m}^{T} \boldsymbol{u}_{m}$. Setting

$$
\boldsymbol{P}^{\frac{1}{2}} \boldsymbol{X}^{T} \boldsymbol{X} \boldsymbol{P}^{\frac{1}{2}} \leqslant \eta \boldsymbol{I}
$$

inequality (33) gives the Lyapunov function limit

$$
\boldsymbol{q}^{T}(t) \boldsymbol{P} \boldsymbol{q}(t) \leqslant 1
$$

and, using Schur complement property, (34) can be rewritten in a closed form as follows

$$
\begin{gathered}
\boldsymbol{X}^{T} \eta^{-1} \boldsymbol{I} \boldsymbol{X} \leqslant \boldsymbol{P}^{-1}=\boldsymbol{Y} \\
{\left[\begin{array}{cc}
\boldsymbol{Y} & \boldsymbol{X}^{T} \\
\boldsymbol{X} & \eta \boldsymbol{I}
\end{array}\right]=\left[\begin{array}{cc}
\boldsymbol{Y} & \boldsymbol{X}^{T} \\
\boldsymbol{X} & \boldsymbol{u}_{m}^{T} \boldsymbol{u}_{m} \boldsymbol{I}
\end{array}\right] \geqslant 0}
\end{gathered}
$$

respectively. An open form of (37) one can obtained using property

$$
\boldsymbol{u}_{m}^{T} \boldsymbol{u}_{m} \boldsymbol{I}=\operatorname{trace}\left(\boldsymbol{u}_{m} \boldsymbol{u}_{m}^{T}\right) \boldsymbol{I} \geqslant \boldsymbol{u}_{m} \boldsymbol{u}_{m}^{T}=\boldsymbol{M}_{r}
$$

Hence, this implies (22).

Using solutions $\gamma>0, Y>0$ and $\boldsymbol{X}$ of this problem, defined by inequalities (21), (22), the controller gain matrix can be found as $\boldsymbol{K}=\boldsymbol{X} \boldsymbol{Y}^{-1}$. This concludes the proof.

\section{Estimator Gain Design}

Theorem 2 Let $\boldsymbol{A}, \boldsymbol{B}, \boldsymbol{M}_{r}$ be given and let $\boldsymbol{K}$ and $\gamma$ be obtained from (21), (22), (23). Then the controlled system with an estimator which is limited in the output and constrained in the input variables is stable if there exist matrices $\mathbb{S}>0, \boldsymbol{V}>0$, and $\mathbf{Z}$ such that it yields

$$
\left[\begin{array}{cc}
\boldsymbol{S}(\boldsymbol{A}-\boldsymbol{B} \boldsymbol{K})+(\boldsymbol{A}-\boldsymbol{B} \boldsymbol{K})^{T} \boldsymbol{S}+\boldsymbol{\gamma}^{-1} \boldsymbol{C}^{T} \boldsymbol{C} & \boldsymbol{S B K} \\
* & \boldsymbol{V A}+\boldsymbol{A}^{T} \boldsymbol{V}-\boldsymbol{Z C}-\boldsymbol{C}^{T} \boldsymbol{Z}^{T}
\end{array}\right]<0
$$




$$
\left[\begin{array}{rrr}
\boldsymbol{S} & \mathbf{0} & \boldsymbol{K}^{T} \\
* & \boldsymbol{V} & -\boldsymbol{K}^{T} \\
* & * & \boldsymbol{M}_{r}
\end{array}\right]>0
$$

where

$$
\boldsymbol{Z}=\boldsymbol{V} \boldsymbol{J} .
$$

Proof Assembling (1), (2) with (5), (6) gives

$$
\left[\begin{array}{c}
\dot{\boldsymbol{q}}(t) \\
\dot{\boldsymbol{q}}_{e}(t)
\end{array}\right]=\left[\begin{array}{cc}
\boldsymbol{A} & -\boldsymbol{B} \boldsymbol{K} \\
\boldsymbol{J C} & \boldsymbol{A}-\boldsymbol{J C}-\boldsymbol{B} \boldsymbol{K}
\end{array}\right]\left[\begin{array}{c}
\boldsymbol{q}(t) \\
\boldsymbol{q}_{e}(t)
\end{array}\right] .
$$

Defining the estimation error vector

$$
\boldsymbol{e}(t)=\boldsymbol{q}(t)-\boldsymbol{q}_{e}(t)
$$

and the congruence transform matrix

$$
\boldsymbol{T}=\boldsymbol{T}^{-1}=\left[\begin{array}{rr}
\boldsymbol{I} & 0 \\
\boldsymbol{I} & -\boldsymbol{I}
\end{array}\right]
$$

then multiplying right-hand side as well as left-hand side of (42) by (44) results in

$$
\begin{gathered}
{\left[\begin{array}{c}
\dot{\boldsymbol{q}}(t) \\
\dot{\boldsymbol{e}}(t)
\end{array}\right]=\left[\begin{array}{cc}
\boldsymbol{A}-\boldsymbol{B} \boldsymbol{K} & \boldsymbol{B K} \\
\boldsymbol{0} & \boldsymbol{A}-\boldsymbol{J C}
\end{array}\right]\left[\begin{array}{l}
\boldsymbol{q}(t) \\
\boldsymbol{e}(t)
\end{array}\right]} \\
\dot{\boldsymbol{q}}^{\bullet}(t)=\boldsymbol{A}^{\bullet} \boldsymbol{q}^{\bullet}(t)
\end{gathered}
$$

respectively, where

$$
\boldsymbol{A}^{\bullet}=\left[\begin{array}{cc}
\boldsymbol{A}-\boldsymbol{B} \boldsymbol{K} & \boldsymbol{B} \boldsymbol{K} \\
\mathbf{0} & \boldsymbol{A}-\boldsymbol{J C}
\end{array}\right], \quad \boldsymbol{q}^{\bullet}(t)=\left[\begin{array}{c}
\boldsymbol{q}(t) \\
\boldsymbol{e}(t)
\end{array}\right] .
$$

Using this extended vector variable $\boldsymbol{q}^{\bullet}(t)$ and defining the partitioned matrix

$$
\boldsymbol{P}^{\bullet}=\boldsymbol{P}^{\bullet T}=\left[\begin{array}{cc}
\boldsymbol{S} & \boldsymbol{W} \\
\boldsymbol{W}^{T} & \boldsymbol{V}
\end{array}\right]>0
$$

then for Lyapunov function of the form

$$
v\left(\boldsymbol{q}^{\bullet}(t)=\boldsymbol{q}^{\bullet T}(t) \boldsymbol{P}^{\bullet} \boldsymbol{q}^{\bullet}(t)>0\right.
$$

it can be easily obtained that the derivative of (49) is

$$
\dot{v}\left(\boldsymbol{q}^{\bullet}(t)\right)=\boldsymbol{q}^{\bullet T}(t)\left(\boldsymbol{A}^{\bullet T} \boldsymbol{P}^{\bullet}+\boldsymbol{P}^{\bullet} \boldsymbol{A}^{\bullet}\right) \boldsymbol{q}^{\bullet}(t)<0 .
$$


With the same output constraints as were defined in (8) the equivalent form of Lyapunov function derivative is

$$
\dot{\mathrm{v}}\left(\boldsymbol{q}^{\bullet}(t)=\boldsymbol{q}^{\bullet T}(t)\left(\boldsymbol{A}^{\bullet T} \boldsymbol{P}^{\bullet}+\boldsymbol{P}^{\bullet} \boldsymbol{A}^{\bullet}\right) \boldsymbol{q}^{\bullet}(t)+\boldsymbol{\gamma}^{-1} \boldsymbol{y}^{T}(t) \boldsymbol{y}(t)-\boldsymbol{\gamma}^{-1} \boldsymbol{y}^{T}(0) \boldsymbol{y}(0)<0 .\right.
$$

Since the generalized weighting matrix $\boldsymbol{P}^{\diamond}$ can be rewritten as follows

$$
\boldsymbol{P}^{\diamond}=\boldsymbol{A}^{\bullet T} \boldsymbol{P}^{\bullet}+\boldsymbol{P}^{\bullet} \boldsymbol{A}^{\bullet}=\left(\boldsymbol{A}_{1}^{\bullet T}+\boldsymbol{A}_{2}^{\bullet T}\right) \boldsymbol{P}^{\bullet}+\boldsymbol{P}^{\bullet}\left(\boldsymbol{A}_{1}^{\bullet}+\boldsymbol{A}_{2}^{\bullet}\right)+\boldsymbol{P}_{Y}^{\bullet}<0
$$

where

$$
\boldsymbol{A}_{1}^{\bullet}=\left[\begin{array}{cc}
\boldsymbol{A}-\boldsymbol{B} \boldsymbol{K} & \boldsymbol{B} \boldsymbol{K} \\
\mathbf{0} & \boldsymbol{A}
\end{array}\right], \quad \boldsymbol{A}_{2}^{\bullet}=\left[\begin{array}{cc}
\mathbf{0} & \mathbf{0} \\
\mathbf{0} & -\boldsymbol{J C}
\end{array}\right], \quad \boldsymbol{P}_{Y}^{\bullet}=\left[\begin{array}{cc}
\gamma^{-1} \boldsymbol{C}^{T} \boldsymbol{C} & \mathbf{0} \\
\mathbf{0} & \mathbf{0}
\end{array}\right]
$$

respectively, then it can be obtained

$$
\boldsymbol{P}^{\bullet} \boldsymbol{A}_{2}^{\bullet}+\boldsymbol{A}_{2}^{\bullet T} \boldsymbol{P}^{\bullet}=-\left[\begin{array}{cc}
\mathbf{0} & \boldsymbol{W J C} \\
\boldsymbol{C}^{T} \boldsymbol{J}^{T} \boldsymbol{W}^{T} & \boldsymbol{V J C}+\boldsymbol{C}^{T} \boldsymbol{J}^{T} \boldsymbol{V}
\end{array}\right] .
$$

Structure of (50) with (54) cannot be written as an LMI and the substitution taking form (29) cannot be used, since additive rank constraint renders the optimization problem non-convex. One way to solve this problem is based on the degenerative structure of $\boldsymbol{P}$, where

$$
W=0, \quad Z=V J
$$

Therefore,

$$
\begin{gathered}
\boldsymbol{P}^{\bullet} \boldsymbol{A}_{2}^{\bullet}+\boldsymbol{A}_{2}^{\bullet T} \boldsymbol{P}^{\bullet}=-\left[\begin{array}{cc}
\mathbf{0} & \mathbf{0} \\
\mathbf{0} & \boldsymbol{Z C}+\boldsymbol{C}^{T} \boldsymbol{Z}^{T}
\end{array}\right] \\
\boldsymbol{P}^{\bullet} \boldsymbol{A}_{1}^{\bullet}+\boldsymbol{A}_{1}^{\bullet T} \boldsymbol{P}^{\bullet}=\left[\begin{array}{cc}
\boldsymbol{S}(\boldsymbol{A}-\boldsymbol{B K})+(\boldsymbol{A}-\boldsymbol{B K})^{T} \boldsymbol{S} & \boldsymbol{S B K} \\
\boldsymbol{K}^{T} \boldsymbol{B}^{T} \boldsymbol{S} & \boldsymbol{V A}+\boldsymbol{A}^{T} \boldsymbol{V}
\end{array}\right]
\end{gathered}
$$

and (50) can be rewritten as

$$
\dot{v}\left(\boldsymbol{q}^{\bullet}(t)\right) \leqslant \boldsymbol{q}^{\bullet T}(t) \boldsymbol{P}^{\diamond} \boldsymbol{q}^{\bullet}(t)<0
$$

where weighting matrix given in (50) takes form (39).

It is evident, that the input constraints have to be included in the estimator parameter design, too. Writing (3) with (44) as follows

$$
\boldsymbol{u}(t)=-\boldsymbol{K}(\boldsymbol{q}(t)-\boldsymbol{e}(t))=-\left[\begin{array}{ll}
\boldsymbol{K} & -\boldsymbol{K}
\end{array}\right] \boldsymbol{q}^{\bullet}(t)=-\boldsymbol{K}^{\bullet} \boldsymbol{q}^{\bullet}(t)
$$

then it is possible to consider

$$
\left\|\boldsymbol{K}^{\bullet} \boldsymbol{q}^{\bullet}(t)\right\|^{2}=\boldsymbol{u}^{T}(t) \boldsymbol{u}(t) \leqslant \boldsymbol{u}_{m}^{T} \boldsymbol{u}_{m}
$$


and to obtain

$$
\boldsymbol{q}^{\bullet T}(t) \boldsymbol{P}^{\bullet \frac{1}{2}} \boldsymbol{P}^{\bullet-\frac{1}{2}} \boldsymbol{K}^{\bullet T} \boldsymbol{K}^{\bullet} \boldsymbol{P}^{\bullet-\frac{1}{2}} \boldsymbol{P}^{\bullet \frac{1}{2}} \boldsymbol{q}^{\bullet}(t) \leqslant \eta
$$

where

$$
\boldsymbol{K}^{\bullet}=\left[\begin{array}{ll}
\boldsymbol{K} & -\boldsymbol{K}
\end{array}\right], \quad \eta=\boldsymbol{u}_{m}^{T} \boldsymbol{u}_{m} .
$$

Setting

$$
\boldsymbol{P}^{\bullet-\frac{1}{2}} \boldsymbol{K}^{\bullet T} \boldsymbol{K}^{\bullet} \boldsymbol{P}^{\bullet-\frac{1}{2}} \leqslant \eta \boldsymbol{I}_{2 n}
$$

inequality (61) gives the Lyapunov function limit

$$
\boldsymbol{q}^{\bullet T}(t) \boldsymbol{P}^{\bullet} \boldsymbol{q}^{\bullet}(t) \leqslant 1 .
$$

Using Schur complement property (63) can now be rewritten in the closed form as follows

$$
\begin{gathered}
\boldsymbol{K}^{\bullet T} \eta^{-1} \boldsymbol{K}^{\bullet} \leqslant \boldsymbol{P}^{\bullet} \\
{\left[\begin{array}{cc}
\boldsymbol{P}^{\bullet} & \boldsymbol{K}^{\bullet T} \\
\boldsymbol{K}^{\bullet} & \eta \boldsymbol{I}_{r}
\end{array}\right]=\left[\begin{array}{cc}
\boldsymbol{P}^{\bullet} & \boldsymbol{K}^{\bullet T} \\
\boldsymbol{K}^{\bullet} & \boldsymbol{u}_{m}^{T} \boldsymbol{u}_{m} \boldsymbol{I}_{r}
\end{array}\right] \geqslant 0} \\
{\left[\begin{array}{cc}
\boldsymbol{P}^{\bullet} & \boldsymbol{K}^{\bullet T} \\
\boldsymbol{K}^{\bullet} & \boldsymbol{M}_{r}
\end{array}\right] \geqslant 0}
\end{gathered}
$$

respectively, which implies (40). This concludes the proof.

Using the solutions $\boldsymbol{V}, \boldsymbol{Z}$ of (39) and (40) the estimator gain matrix can be found as $\boldsymbol{J}=\boldsymbol{V}^{-1} \boldsymbol{Z}$.

Theorem 3 (Unified algebraic approach) Let $\boldsymbol{A}, \boldsymbol{B}, \boldsymbol{M}_{r}$ are given and let $\boldsymbol{K}$ and $\gamma$ are obtained from (21), (22), (23). Then the controlled system with an estimator which is limited in the output and constrained in the input variables is stable if there exist matrices $\boldsymbol{S}>0, \boldsymbol{V}>0$ such that

$$
\begin{aligned}
& {\left[\begin{array}{c}
\mathbf{0} \\
\boldsymbol{C}^{T}
\end{array}\right]^{\perp}\left[\begin{array}{cc}
\boldsymbol{S}(\boldsymbol{A}-\boldsymbol{B K})+(\boldsymbol{A}-\boldsymbol{B} \boldsymbol{K})^{T} \boldsymbol{S}+\boldsymbol{\gamma}^{-1} \boldsymbol{C}^{T} \boldsymbol{C} & \boldsymbol{S B K} \\
* & \boldsymbol{V A}+\boldsymbol{A}^{T} \boldsymbol{V}
\end{array}\right]\left[\begin{array}{c}
\mathbf{0} \\
\boldsymbol{C}^{T}
\end{array}\right]^{\perp T}<0} \\
& {\left[\begin{array}{rrr}
\boldsymbol{S} & \mathbf{0} & \boldsymbol{K}^{T} \\
* & \boldsymbol{V} & -\boldsymbol{K}^{T} \\
* & * & \boldsymbol{M}_{r}
\end{array}\right]>0}
\end{aligned}
$$

Then the estimator gain matrix exists if for obtained $\mathbf{S}, \boldsymbol{V}$ there exist symmetric matrices $\boldsymbol{R}>0, \boldsymbol{N}>0$ such that it yields

$$
\left[\begin{array}{cc}
-\boldsymbol{F} \boldsymbol{R} \boldsymbol{F}^{T}-\boldsymbol{H} & \boldsymbol{F} \boldsymbol{R}+\boldsymbol{G}^{T} \boldsymbol{L}^{T} \\
* & -\boldsymbol{R}
\end{array}\right]<0
$$


where

$$
\begin{gathered}
\boldsymbol{F}=\left[\begin{array}{c}
\mathbf{0} \\
\boldsymbol{C}^{T}
\end{array}\right], \quad \boldsymbol{L}^{T}=\left[\begin{array}{r}
\mathbf{0} \\
-\boldsymbol{J}
\end{array}\right], \quad \boldsymbol{G}=\left[\begin{array}{cc}
\boldsymbol{S} & \mathbf{0} \\
\mathbf{0} & \boldsymbol{V}
\end{array}\right] \\
\boldsymbol{H}=\boldsymbol{N}-\left[\begin{array}{cc}
\boldsymbol{S}(\boldsymbol{A}-\boldsymbol{B} \boldsymbol{K})+(\boldsymbol{A}-\boldsymbol{B} \boldsymbol{K})^{T} \boldsymbol{S}+\boldsymbol{\gamma}^{-1} \boldsymbol{C}^{T} \boldsymbol{C} & \boldsymbol{S B K} \\
* & \boldsymbol{V A}+\boldsymbol{A}^{T} \boldsymbol{V}
\end{array}\right]<0 .
\end{gathered}
$$

Proof. Now inequality (57) can be partitioned as follows

$$
\begin{gathered}
{\left[\begin{array}{cc}
\boldsymbol{S}(\boldsymbol{A}-\boldsymbol{B} \boldsymbol{K})+(\boldsymbol{A}-\boldsymbol{B} \boldsymbol{K})^{T} \boldsymbol{S}+\boldsymbol{\gamma}^{-1} \boldsymbol{C}^{T} \boldsymbol{C} & \boldsymbol{S B K} \\
\boldsymbol{K}^{T} \boldsymbol{B}^{T} \boldsymbol{S} & \boldsymbol{V A}+\boldsymbol{A}^{T} \boldsymbol{V}
\end{array}\right]+} \\
+\left[\begin{array}{c}
\mathbf{0} \\
\boldsymbol{C}^{T}
\end{array}\right]\left[\begin{array}{ll}
\mathbf{0} & -\boldsymbol{J}^{T}
\end{array}\right]\left[\begin{array}{ll}
\boldsymbol{S} & \mathbf{0} \\
\mathbf{0} & \boldsymbol{V}
\end{array}\right]+\left[\begin{array}{cc}
\boldsymbol{S} & \mathbf{0} \\
\mathbf{0} & \boldsymbol{V}
\end{array}\right]\left[\begin{array}{c}
\mathbf{0} \\
-\boldsymbol{J}
\end{array}\right]\left[\begin{array}{ll}
\mathbf{0} & \boldsymbol{C}
\end{array}\right]<0 .
\end{gathered}
$$

Using the orthogonal complement

$$
\boldsymbol{C}^{\bullet T \perp}=\left[\begin{array}{c}
\mathbf{0} \\
\boldsymbol{C}^{T}
\end{array}\right]^{\perp}
$$

then pre-multiplying left-hand side of (73) by (74), and right-hand side of (73) by the transposition of (74) leads to the inequality

$$
\left[\begin{array}{c}
\mathbf{0} \\
\boldsymbol{C}^{T}
\end{array}\right]^{\perp}\left[\begin{array}{cc}
\boldsymbol{S}(\boldsymbol{A}-\boldsymbol{B K})+(\boldsymbol{A}-\boldsymbol{B K})^{T} \boldsymbol{S}+\boldsymbol{\gamma}^{-1} \boldsymbol{C}^{T} \boldsymbol{C} & \boldsymbol{S B K} \\
\boldsymbol{K}^{T} \boldsymbol{B}^{T} \boldsymbol{S} & \boldsymbol{V A}+\boldsymbol{A}^{T} \boldsymbol{V}
\end{array}\right]\left[\begin{array}{c}
\mathbf{0} \\
\boldsymbol{C}^{T}
\end{array}\right]^{\perp T}<0 .
$$

In the sense of (73) one can set

$$
\boldsymbol{F} \boldsymbol{L} \boldsymbol{G}+\boldsymbol{G}^{T} \boldsymbol{L}^{T} \boldsymbol{F}^{T}-\boldsymbol{H}<-\boldsymbol{N}
$$

where $N=N^{T}>0$

$$
\begin{gathered}
\boldsymbol{F}=\left[\begin{array}{c}
\mathbf{0} \\
\boldsymbol{C}^{T}
\end{array}\right], \quad \boldsymbol{L}^{T}=\left[\begin{array}{r}
\mathbf{0} \\
-\boldsymbol{J}
\end{array}\right], \quad \boldsymbol{G}=\left[\begin{array}{cc}
\boldsymbol{S} & \mathbf{0} \\
\mathbf{0} & \boldsymbol{V}
\end{array}\right] \\
\boldsymbol{H}=\boldsymbol{N}-\left[\begin{array}{cc}
\boldsymbol{S}(\boldsymbol{A}-\boldsymbol{B} \boldsymbol{K})+(\boldsymbol{A}-\boldsymbol{B K})^{T} \boldsymbol{S}+\boldsymbol{\gamma}^{-1} \boldsymbol{C}^{T} \boldsymbol{C} & \boldsymbol{S B K} \\
\boldsymbol{K}^{T} \boldsymbol{B}^{T} \boldsymbol{S} & \boldsymbol{V A}+\boldsymbol{A}^{T} \boldsymbol{V}
\end{array}\right]<0 .
\end{gathered}
$$

Then there exists a matrix $\boldsymbol{R}>0$ such, that

$$
\boldsymbol{F} \boldsymbol{L} \boldsymbol{G}+\boldsymbol{G}^{T} \boldsymbol{L}^{T} \boldsymbol{F}^{T}-\boldsymbol{H}+\boldsymbol{G}^{T} \boldsymbol{L}^{T} \boldsymbol{R}^{-1} \boldsymbol{L} \boldsymbol{G}<0 .
$$

After completing the square it can be obtained

$$
\left(\boldsymbol{F} \boldsymbol{R}+\boldsymbol{G}^{T} \boldsymbol{L}^{T}\right) \boldsymbol{R}^{-1}\left(\boldsymbol{F} \boldsymbol{R}+\boldsymbol{G}^{T} \boldsymbol{L}^{T}\right)^{T}-\boldsymbol{F} \boldsymbol{R} \boldsymbol{F}^{T}-\boldsymbol{H}<0
$$


and any solution $\boldsymbol{L}$ of (80) exists if there exist symmetric positive definite matrices $\mathbb{R}>0$, $N>0$ such that the LMI

$$
\left[\begin{array}{cc}
-\boldsymbol{F} \boldsymbol{R} \boldsymbol{F}^{T}-\boldsymbol{H} & \boldsymbol{F} \boldsymbol{R}+\boldsymbol{G}^{T} \boldsymbol{L}^{T} \\
* & -\boldsymbol{R}
\end{array}\right]<0
$$

is satisfied. This concludes the proof.

Specially it is possible to define (74) as follows

$$
\boldsymbol{C}^{\bullet T \perp}=\left[\begin{array}{c}
0 \\
\boldsymbol{C}^{T}
\end{array}\right]^{\perp}=\left[\begin{array}{ll}
\boldsymbol{C}^{T \perp} & \boldsymbol{C}^{T \perp}
\end{array}\right] .
$$

Then (75) implies

$$
\boldsymbol{C}^{T \perp}\left(\boldsymbol{S}\left(\boldsymbol{A}+\boldsymbol{A}^{T}\right) \boldsymbol{S}+\boldsymbol{V}\left(\boldsymbol{A}+\boldsymbol{A}^{T}\right) \boldsymbol{V}\right) \boldsymbol{C}^{T \perp T}<0
$$

which gives the condition

$$
S=V
$$

These can be formulated as the corollary.

Corollary 1 (Separability principle) Let $\boldsymbol{A}, \boldsymbol{B}, \boldsymbol{M}_{r}$ be given, and let $\boldsymbol{K}$ and $\gamma$ are obtained from (21), (22), (23). Then the controlled system with an estimator which is limited in the output and constrained in the input variables is stable if there exists a matrix $\mathbf{S}>0$ such that

$$
\begin{gathered}
\boldsymbol{C}^{T \perp} \boldsymbol{S}\left(\boldsymbol{A}+\boldsymbol{A}^{T}\right) \boldsymbol{S} \boldsymbol{C}^{T \perp T}<0 \\
{\left[\begin{array}{ccc}
\boldsymbol{S} & \mathbf{0} & \boldsymbol{K}^{T} \\
* & \boldsymbol{S} & -\boldsymbol{K}^{T} \\
* & * & \boldsymbol{M}_{r}
\end{array}\right]>0 .}
\end{gathered}
$$

Then the estimator gain matrix exists if for obtained $\mathbf{S}$ there exist symmetric matrices $\boldsymbol{R}>0, \boldsymbol{N}>0$ such that it yields (70) through (72) with $\boldsymbol{G}=\operatorname{diag}[\boldsymbol{S} \boldsymbol{S}]$.

Knowing once $L$ one can obtain $J$.

\section{Illustrative example}

The system was given by (1), (2), where

$$
\boldsymbol{A}=\left[\begin{array}{rrr}
0 & 1 & 0 \\
0 & 0 & 1 \\
-5 & -9 & -5
\end{array}\right], \quad \boldsymbol{B}=\left[\begin{array}{ll}
0 & 0 \\
1 & 1 \\
1 & 0
\end{array}\right], \quad \boldsymbol{C}^{T}=\left[\begin{array}{ll}
1 & 1 \\
2 & 1 \\
1 & 0
\end{array}\right], \quad \boldsymbol{u}_{m}^{T}=\left[\begin{array}{l}
0.5 \\
0.01
\end{array}\right]
$$


Solving (21), (22) for LMI matrix variables $\boldsymbol{\gamma}>0, \boldsymbol{Y}>0, \boldsymbol{X}$ using SeDuMi (Self-DualMinimization) package for Matlab [16] the feedback gain matrix design was feasible with

$$
\begin{gathered}
\gamma=0.4725 \\
\boldsymbol{Y}=\left[\begin{array}{rrr}
0.3045 & -0.1586 & -0.0595 \\
-0.1586 & 0.1981 & -0.1090 \\
-0.0595 & -0.1090 & 0.3355
\end{array}\right], \quad \boldsymbol{X}=\left[\begin{array}{lll}
0.0448 & 0.0670 & -0.1316 \\
0.0009 & 0.0013 & -0.0026
\end{array}\right]
\end{gathered}
$$

to give the design parameter as follows

$$
\begin{gathered}
\boldsymbol{K}=\left[\begin{array}{lll}
0.4838 & 0.6784 & -0.0861 \\
0.0097 & 0.0136 & -0.0017
\end{array}\right] \\
\rho(\boldsymbol{A}-\boldsymbol{B} \boldsymbol{K})=\{-0.7903,-2.4078 \pm \mathrm{i} 2.1951\} .
\end{gathered}
$$

It is evident, that the eigenvalues spectrum of the closed control loop is stable.

Now solving (85), (86) it was obtained

$$
\boldsymbol{S}=\boldsymbol{V}=\left[\begin{array}{lll}
6.3661 & 2.9359 & 0.4581 \\
2.9359 & 9.5564 & 0.5212 \\
0.4581 & 0.5212 & 1.0022
\end{array}\right]
$$

Constructing (72) for $\gamma, \boldsymbol{K}, \boldsymbol{S}, \boldsymbol{V}$, and $\boldsymbol{N}=5 \boldsymbol{I}_{2 n}$, and solving inequality (70) as the feasible problem with $R=0.1 \boldsymbol{I}$ these results were found immediately

$$
\begin{gathered}
\boldsymbol{L}^{T}=\left[\begin{array}{rrrrrr}
0 & 0 & 0 & -0.0024 & -0.0152 & -0.0896 \\
0 & 0 & 0 & -0.0134 & -0.0068 & 0.0093
\end{array}\right] \\
\boldsymbol{J}^{T}=\left[\begin{array}{rrr}
-0.0024 & -0.0152 & -0.0896 \\
-0.0134 & -0.0068 & 0.0093
\end{array}\right] \\
\rho(\boldsymbol{A}-\boldsymbol{J} \boldsymbol{C})=\{-1.0000,-1.9287 \pm \mathrm{i} 1.0567\} .
\end{gathered}
$$

It can be seeing that the state estimator is stable, too.

\section{Concluding remarks}

The paper presents one new method to design the state estimator parameters for known controller gain matrix and the prescribed input variables constraints. The method is based on the unified algebraic approach using LMI principle and can easily be modified for the discrete-time system models. 


\section{References}

[1] G. BesanCon (ED.): Nonlinear observers and applications. Springer, Berlin, 2007.

[2] M. Blanke, M. Kinnaert, J. Lunze and M. Staroswiecki: Diagnosis and fault-tolerant control. Springer, Berlin, 2003.

[3] D. Boyd, L. El Ghaoui, E. Peron and V. Balakrishnan: Linear matrix inequalities in system and control theory. SIAM, Philadelphia, 1994.

[4] S.X. Ding: Model-based fault diagnosis techniques. Design Schemes, Algorithms, and Tools. Springer, Berlin, 2008.

[5] A. Filasová and D. KROKAVEC: LMI-supported design of residual generators based on unknown-input estimator scheme. Prep. of the $\sigma^{h}$ IFAC Symp. on Robust Control Design ROCOND '09., Haifa, Israel, (2009), 313-319.

[6] P. Gahinet, A. Nemirovski, A.J. Laub and M. Chilali: LMI control toolbox user's guide. The MathWorks, Inc., Natick, 1995.

[7] R.E. Kalman: A new approach to linear filtering and prediction problems. Trans. of the ASME, Series D, Journal of Basic Engineering, 82 (1960), 35-45.

[8] D. Krokavec and A. Filasová: Dynamic systems diagnosis. Elfa, Košice, 2007, (in Slovak).

[9] D. Krokavec and A. Filasová: Discrete-time systems. Elfa, Košice, 2008, (in Slovak).

[10] H. Lens and J. ADAmY: Observed based controller design for linear systems with input constraints. Proc. of the $17^{\text {th }}$ World Congress IFAC 2008, Seoul, Korea, (2008), 1243-1248.

[11] D.G. LuenBerg: Observing the state of a linear system IEEE Trans. on Military Electronics, MIL-8 (1964), 74-80.

[12] M.S. MAhmoud: Resilient control of uncertain dynamical systems. Springer, Berlin, 2004.

[13] Y. Nesterov and A. Nemirovsky: Interior-point polynomial methods in convex programming, SIAM, Philadelphia, 1994.

[14] E.G. Nobrega, M.O. Abdalla and K.M. Grigoriadis: LMI-based filter design for fault detection and isolation. Proc. of the $39^{h}$ IEEE Conf. on Decision and Control, Sydney, Australia, 5 (2000), 4329-4334.

[15] J. O’ReIlly: Observers for linear systems. Academic Press, London, 1983. 
[16] D. Paucelle, D. Henrion, Y. Labit and K. Taitz: User's guide for SeDuMi interface 1.04. LAAS-CNRS, Toulouse, 2002.

[17] D. Simon: Optimal state estimation: Kalman, $\mathrm{H}_{\infty}$ and nonlinear approaches. John Wiley \& Sons, Inc., Hoboken, 2006.

[18] R.E. Skelton, T. IVASAKI and K. Grigoriadis: A unified algebraic approach to linear control design. Taylor \& Francis, London, 1998.

[19] X. Zhan: Matrix inequalities. Springer, Berlin, 2002. 